\title{
Carbapenem Resistant Enterobacteriaceae infections in Houston, Texas: An outline
}

\author{
Hafeez Rehman*, Imran Shaikh, Kasimu Muhetaer and Salma Khuwaja \\ Houston Health Department, Houston, TX, USA
}

\section{Objective}

To examine demographic as well as clinical characteristics of the Carbapenam Resistant Enteriobacteriacae (CRE) Organisms cases in Houston, Texas, 2015-2016

\section{Introduction}

According to CDC, CRE is used to describe bacteria that are nonsusceptible to one or more carbapenems; doripenem, meropenem or imipenem and resistant to third generation cephalosporins like ceftriaxone, cefotaxime and ceftazidime. These organisms cause infections that are associated with high mortality rates and they have the potential to spread widely. Antibiotic resistant bacteria cause more than 2 million illnesses and at least 23,000 deaths each year in United States. CREs are found in many health care settings like acute care hospitals, long term care facilities, nursing homes, rehabilitation facilities and other health care settings. Although CREs includes a number of species, reporting in State of Texas is limited to CREKlebsiella species and CRE-E.coli.

\section{Methods}

Population-based surveillance data was generated from Houston's electronic disease surveillance system reported to Houston Health Department (HHD) from October 2015 to July 2016. Descriptive analysis was performed to examine demographic and clinical characteristics across different age groups, gender and race/ethnicity. HHD has received a total of 463 CRE cases during the time period, out of which 72 were non-reportable and did not meet the case criteria, 187 were out of jurisdiction. The remaining 204 cases were included in this study.

\section{Results}

Out of a total of 204 cases, males and females were represented equally $(50 \%$ each). The mean age of the cases was 67 years (age ranges from 22-98). Majority of the cases were in the older age group, 70 years and above $53(26 \%)$, followed by 48 (24\%) in age group 80 and above years. Among the different race/ethnic groups, African-Americans comprised of $82(40 \%)$, followed by Whites $67(33 \%)$ and Hispanics $33(16 \%)$. Out of 204 cases, $156(76 \%)$ were hospitalized, which included acute care hospital, long-term acute care or nursing home. Out of 156 hospitalized cases, 71 (34\%) were in Intensive Care Unit (ICU) and $136(67 \%)$ had an invasive or indwelling device. Of all the cases, $80 \%$ had CRE Klebsiella pneumoniae, followed by $11 \%$ who had CRE- E coli. The cases were distributed evenly across the city when plotted on ArcGIS with their residential addresses.

\section{Conclusions}

CRE cases are found to be more common among older age groups, African American population and in hospitalized patients. CRE can be a ground for increasing infectious diseases in the community and one of the reason may be unnecessary use of antimicrobial agents. This study provides a glimpse into the number of CRE cases reported in Houston since CREs are classified a separate disease in Texas. Further studies are needed to explore the occurrence of anti-microbial drug resistance among the specific population groups and how the case investigation efforts can be targeted to enhance prevention.
Keywords

CRE; Drug resistant; Anti-biotics

Acknowledgments

Staff of the Bureau of Epidemiology, Houston Health Department

*Hafeez Rehman

E-mail: Hafeez.Rehman@houstontx.gov 